\title{
Reversible helical unwinding transition of a self-assembling peptide amphiphile $\dagger$
}

Cite this: Soft Matter, 2013, 9, 9290

Received 25th June 2013

Accepted 19th August 2013

DOI: $10.1039 / c 3 s m 51725 j$

www.rsc.org/softmatter

A designed peptide amphiphile $\mathrm{C}_{16}$-KKFFVLK self-assembles into nanotubes and helical ribbons in aqueous solution at room temperature. A remarkable unwinding transition, leading to twisted tapes, is observed on heating. Nanotubes and ribbons re-form on cooling.

The formation of nanotubes by self-assembling systems continues to fascinate. ${ }^{1}$ Chiral self-assembly of twisted sheets and helical ribbons has also been studied for systems as diverse as seed pods, ${ }^{2}$ hexabenzocoronene derivatives, ${ }^{3}$ zinc oxide nanobelts, ${ }^{4}$ bile salts, ${ }^{5}$ gemini surfactants ${ }^{6}$ and peptides ${ }^{7}$ among others. The closure of helical ribbons is a possible mechanism for the formation of nanotubes, as suggested by experiments, ${ }^{5,6 \boldsymbol{6}, 7 \boldsymbol{b}}$ and various theories. ${ }^{8}$ Recently, "pulsating" tubules have been observed where the size of chiral tubules formed by noncovalent macrocycles can be switched thermally. ${ }^{9}$

We have discovered that a peptide amphiphile undergoes a reversible thermal transition between a state of predominantly nanotubes and helical ribbons and twisted tapes at higher temperature. Here, the nature of this remarkable transition is elucidated using a combination of microscopy, X-ray scattering and spectroscopic methods.

The peptide amphiphile (PA) has been designed based on the KLVFF core motif from the amyloid beta $(\mathrm{A} \beta)$ peptide. ${ }^{10}$ In the KKFFVLK heptapeptide sequence exploited here, the two $\mathrm{F}$ residues in KKFFVLK drive self-assembly through hydrophobic and $\pi$-stacking interactions. ${ }^{7 \boldsymbol{a}, \boldsymbol{b}, \mathbf{1 1}}$ Two additional lysine residues are incorporated between the hexadecanyl lipid chain and the KKFFVLK motif to promote solubility and impart

${ }^{a}$ Dept of Chemistry, University of Reading, Whiteknights, Reading RG6 6AD, UK. E-mail: I.W.Hamley@reading.ac.uk

${ }^{b}$ Colworth Discover-Unilever R\&D, Sharnbrook MK44 1LQ, UK

${ }^{c}$ Dept of Applied Physics, Aalto University School of Science, PO Box 15100, FI-00076 Aalto, Finland

$\dagger$ Electronic supplementary information (ESI) available: Experimental methods and characterization data. Additional TEM, SAXS, FTIR and POM data, and critical aggregation concentration data. See DOI: 10.1039/c3sm51725j amphiphilicity. This communication complements our recent report on the enzymatic degradation of this PA. ${ }^{12}$

Cryogenic transmission electron microscopy (cryo-TEM) is a powerful tool to image self-assembled structures, via cryovitrification of the aqueous medium. Fig. $1 \mathrm{a}$ and $\mathrm{b}$ show cryoTEM images showing nanotubes of $\mathrm{C}_{16}$-KKFFVLK coexisting with helical ribbons at room temperature. The tube walls are extremely thin, less than $5 \mathrm{~nm}$ as indicated by images such as Fig. 1b. Fig. 1c and ESI Fig. $1 \dagger$ show twisted tapes which are the predominant structure at $55{ }^{\circ} \mathrm{C}$. Additional cryo-TEM images are presented in ESI Fig. $1 . \dagger$

Small-angle X-ray scattering (SAXS) was used to further probe the in situ self-assembled nanostructure. Fig. 2 presents data measured at $20{ }^{\circ} \mathrm{C}$ and $55^{\circ} \mathrm{C}$. The data at $20^{\circ} \mathrm{C}$ can be fitted to a
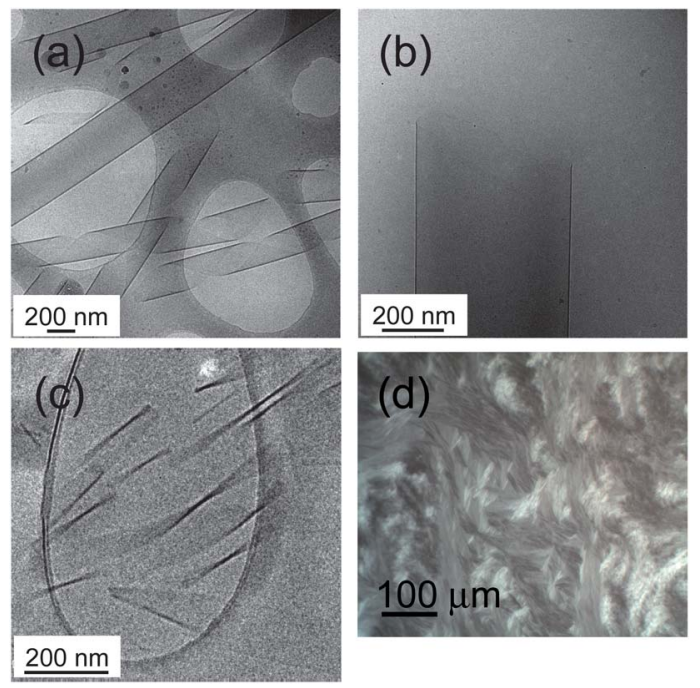

Fig. 1 (a-c) Cryo-TEM images showing nanostructures from self-assembly of $\mathrm{C}_{16}$-FFKLVFF (1 wt\%, i.e. $87 \mathrm{mM}$, solution) (a) single wall nanotubes coexisting with helical ribbons at $22{ }^{\circ} \mathrm{C}$, (b) single wall nanotube at $22{ }^{\circ} \mathrm{C}$, showing thin wall, (c) twisted tapes at $55^{\circ} \mathrm{C}$. (d) Birefringence texture in a polarized optical micrograph of a $2 \mathrm{wt} \%$ sample at $22{ }^{\circ} \mathrm{C}$. 

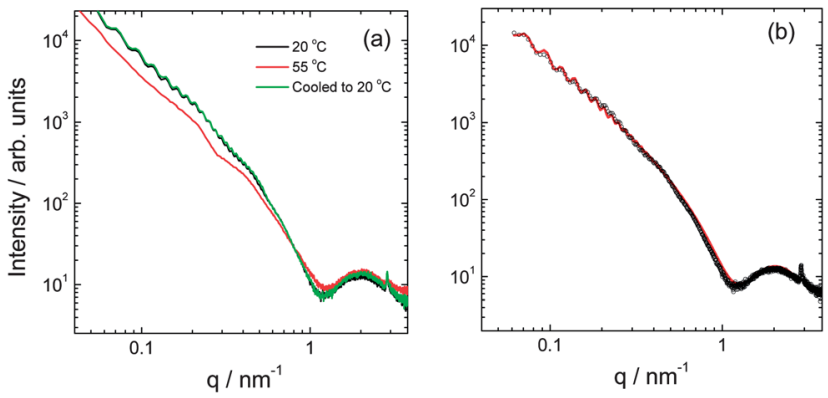

Fig. 2 (a) SAXS data for a $1 \mathrm{wt} \%$ solution obtained at $20^{\circ} \mathrm{C}$, after heating to $55^{\circ} \mathrm{C}$ and after cooling back to $20^{\circ} \mathrm{C}$. (b) Form factor fit (red line) using a nanotube model to fit the data (open circles) at $20^{\circ} \mathrm{C}$

model form factor that consists of two contributions, one from nanotubes and the other from "lipid-like" bilayer structures. The fit indicates a nanotube radius $R=138 \mathrm{~nm}$ (with a very low polydispersity $2 \%$ ) and a wall thickness $4.4 \mathrm{~nm}$. The obtained nanotube radius is in good agreement with that estimated from cryo-TEM.

On heating to $55{ }^{\circ} \mathrm{C}$, the nanotube form factor oscillations disappear, leaving essentially a form factor profile from the helically wrapped PA bilayers. However, the nanotube form factor oscillations re-appear on cooling. The kinetics of this process was monitored, indicating that this process takes up to several hours, depending on the incubation time at $55^{\circ} \mathrm{C}$ and the cooling rate.

The nanotubes exhibit spontaneous flow-induced alignment, as revealed by SAXS (Fig. 3a). This suggests nematic orientational ordering. This was confirmed by polarized optical microscopy (Fig. 1d and ESI Fig. 2, $\dagger$ showing thermo-reversibility of birefringence when the sample was heated to $55^{\circ} \mathrm{C}$ and then cooled back to $22^{\circ} \mathrm{C}$ ). By SAXS, alignment was observed on flowing the sample through the capillary holder, and the extent of orientation could be increased by manually shearing by driving a delivery syringe back and forth. SAXS shows that orientation of the sample is retained on heating to $55{ }^{\circ} \mathrm{C}$ (ESI Fig. $3 \dagger$ ), although alignment with respect to the flow direction is lost in the absence of shear forces. The form factor oscillations
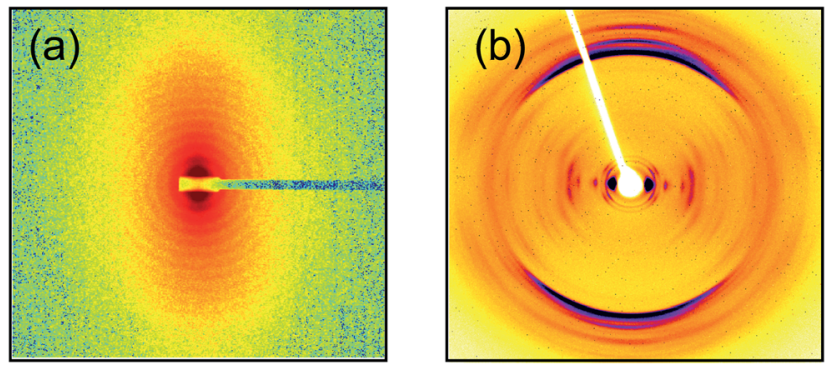

Fig. 3 (a) SAXS data for a flow-aligned sample of $1 \mathrm{wt} \% \mathrm{C}_{16}-\mathrm{KKFFVLKFF}$ at $20^{\circ} \mathrm{C}$, $q$ scale: -0.3 to $0.3 \mathrm{~nm}^{-1}$. The flow direction is horizontal. The intensity is contoured on a logarithmic scale. (b) X-ray diffraction pattern obtained from a stalk dried from a 1 wt\% sample (linear intensity scale). The fibril axis is vertical. The strong meridional reflection has a spacing $4.88 \AA$, and the spacing of other strong reflections is discussed in the text. disappear (consistent with the 1D data in Fig. 2a). The extent of orientation as gauged by the anisotropy of the SAXS pattern appears greater at $55^{\circ} \mathrm{C}$ (compare ESI Fig. $3 \mathrm{~b}$ and a or $\mathrm{d} \dagger$ ). On cooling to $20{ }^{\circ} \mathrm{C}$, the form factor oscillations reappear within 30 minutes, although they continue to develop for up to $9 \mathrm{~h}$ (ESI Fig. $3 \dagger$ ).

X-ray diffraction on stalks prepared by drying also shows strong orientation, as well as features arising from the helical arrangement of the peptide strands within the tube wall (Fig. 3b). In particular, the prominent near-equatorial reflections with $d=2.68 \mathrm{~nm}, d=2.25 \mathrm{~nm}$ and $d=1.09 \mathrm{~nm}$ are split off-axis, as are several weaker peaks with smaller $d$ spacings. These coexist with on-axis equatorial reflections with $d=3.65$, 1.87 and $1.23 \mathrm{~nm}$. These correspond to the first, second and third order reflections respectively of a layered structure with spacing $d=3.7 \mathrm{~nm}$. This value is very close (considering dehydration upon drying) to the nanotube wall thickness estimated from the fit to the SAXS form factor (Fig. 2a, actually it can be estimated from the broad maximum near $q=2 \mathrm{~nm}^{-1}$ ) and indicates that upon drying the sample for XRD, either the nanotubes collapse to give a multi-layer stack, or there is an increase in the population of multi-wall nanotubes. The $d=$ $2.25 \mathrm{~nm}$ reflection spacing is very similar to that of the sharp Bragg peak in the SAXS data (Fig. 2) with $d=2.2 \mathrm{~nm}$. The XRD pattern in Fig. 3b also features strong meridional reflections with $d=4.88 \AA$ (v. strong) and $4.52 \AA$ (strong) and $4.14 \AA$ (strong). The main $d$ spacing is that expected for the spacing of $\beta$-strands in a $\beta$-sheet structure. Further evidence for $\beta$-sheet structure is provided by FTIR (ESI Fig. $4 \dagger$ ) which shows a peak in the amide I' region at $1625 \mathrm{~cm}^{-1} \cdot{ }^{13}$ In addition, the uptake of thioflavin $\mathrm{T}$ (ThT) is observed for $\mathrm{C}_{16}$-KKFFVLK aggregates, consistent with the formation of "amyloid" $\beta$-sheet structures. ${ }^{\mathbf{1 4}}$ As shown in ESI Fig. $5, \uparrow$ this technique was used to determine a low critical aggregation concentration (cac) of $0.012 \mathrm{wt} \%$. The dye ThT is taken up selectively by $\beta$-sheet amyloid structures ${ }^{\mathbf{1 0 c}, \mathbf{1 5}}$ however the mechanism of binding to amyloid has not been definitively identified. While some papers suggest ThT binds to $\beta$-sheet grooves, other research provides some evidence that the binding is dependent on electrostatic interactions or diameter-dependent binding to molecular grooves/ cavities in $\beta$-sheet and other fibers. ${ }^{16}$ Electrostatic interactions between the cationic dye and the cationic PA here should hinder binding, although counterion effects may screen charge leading to predominant "steric" binding. The cac results show that at the concentrations at which self-assembly was studied by X-ray scattering and electron microscopy, the PA will be strongly aggregated.

The X-ray scattering data lead to a model in which the PA molecules form a highly interdigitated bilayer $4.4 \mathrm{~nm}$ thick. The estimated length of each $\mathrm{C}_{16}$-KKFFVLK molecule is $4 \mathrm{~nm}$, assuming $3.2 \AA$ per peptide residue in a parallel $\beta$-sheet. ${ }^{17}$ ESI Fig. $4 \dagger$ shows FTIR spectra which are consistent with this secondary structure (antiparallel $\beta$-sheets have an additional peak in the $1680-1690 \mathrm{~cm}^{-1}$ range). ${ }^{13 b, 18}$ Based on this estimation, it appears that only the C-terminal lysine protrudes from the lipid-rich hydrophobic bilayer core, in which the KKFFVL residues are also buried. The $\mathrm{C}_{16}$-KKFFVLK 
nanotubes are therefore expected to comprise lysine-rich surfaces.

The thermoreversible unwinding of the helical ribbons and nanotubes into twisted tapes (Fig. 4) involves a change in curvature of the PA bilayer. This may be driven by temperaturedependent changes in peptide solubility which may also be influence by thermally-induced changes in hydrogen bonding strength, both within the peptide $\beta$-sheets and also with the water solvent molecules. A role of $\mathrm{pH}$ is unlikely, since the $\mathrm{pH}$ does not change much from 2.89 at $20{ }^{\circ} \mathrm{C}$ to 2.73 at $55{ }^{\circ} \mathrm{C}$ for a $1 \mathrm{wt} \%$ solution. Estimates of the interaction energies within folding proteins suggest that aromatic stacking interaction energies are larger than those associated with hydrogen bonds or salt bridges ${ }^{\mathbf{1 9}}$ and the $\mathrm{F}-\mathrm{F}$ interactions here are likely to be significant. Consistent with this, CD spectra reveal remarkable pronounced temperature-dependent and reversible changes in the vibronic structure associated with phenylalanine (Fig. 5). The strong features in the CD spectra reflect the strongly chiral environment of the two $\mathrm{F}$ residues. The reduction in the magnitude of the ellipticity above $255 \mathrm{~nm}$ at $55{ }^{\circ} \mathrm{C}$ is consistent with the lower chirality of the unwrapped tapes. The CD spectra in the region sensitive to secondary structure are reported in our recent paper, ${ }^{12}$ unfortunately absorbance issues hindered temperature-dependent measurements in this region. Nevertheless, CD points to a likely role of changes in phenylalanine stacking as a driver for the observed transition.

From a phenomenological perspective, the transition between twisted tapes and ribbons/nanotubes can be analysed using models for the elasticity of chiral membranes, ${ }^{6 a}$ although

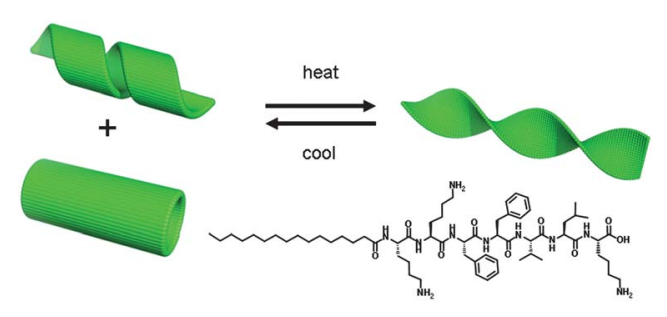

Fig. 4 Schematic of the thermo-reversible transition, and (bottom right) structure of the peptide amphiphile.

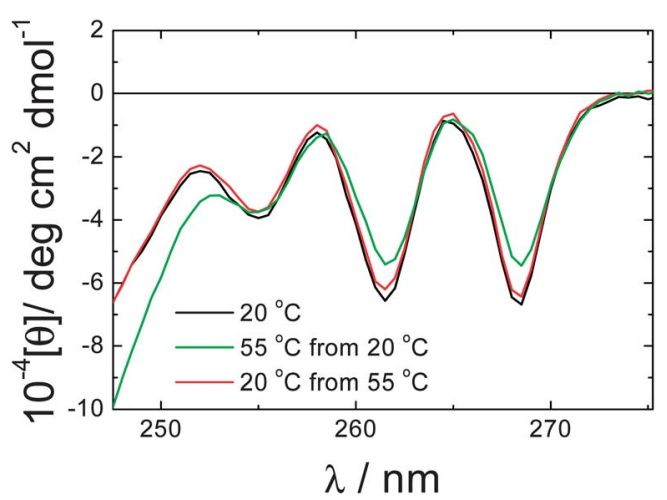

Fig. 5 Circular dichroism spectra obtained from a 1 wt\% solution of $\mathrm{C}_{16^{-}}$ KKFFVLK at the temperatures indicated. quantification would require estimates of the bending stiffness among other parameters which are not currently available for our system.

The thermally-induced unraveling of the nanotubes suggests possible applications in the release of encapsulated cargo. This might be useful in therapeutic applications, where the cationic functionality of the nanotube surface could be employed in targeted delivery. There also exists the potential to exploit the longitudinal expansion and contraction of the fibrillar structure that occurs when the helical ribbons unwind or refold upon heating/cooling. This could be localized if a local heat source (e.g. chemical reaction) was available. These and other aspects will be the subject of future research.

\section{Notes and references}

1 T. Shimizu, M. Masuda and H. Minamikawa, Chem. Rev., 2005, 105, 1401-1443.

2 S. Armon, E. Efrati, R. Kupferman and E. Sharon, Science, 2011, 333, 1726-1730.

3 W. Zhang, W. Jin, T. Fukushima, N. Ishii and T. Aida, J. Am. Chem. Soc., 2013, 135, 114-117.

4 P. X. Gao, Y. Ding, W. J. Mai, W. L. Hughes, C. S. Lao and Z. L. Wang, Science, 2005, 309, 1700-1704.

5 P. Terech, S. K. P. Velu, P. Pernot and L. Wiegart, J. Phys. Chem. B, 2012, 116, 11344-11355.

6 (a) R. Oda, I. Huc, M. Schmutz, S. J. Candau and F. C. MacKintosh, Nature, 1999, 399, 566-569; (b) L. Ziserman, H. Y. Lee, S. R. Raghavan, A. Mor and D. Danino, J. Am. Chem. Soc., 2011, 133, 2511-2517; (c) L. Ziserman, A. Mor, D. Harries and D. Danino, Phys. Rev. Lett., 2011, 106, 238105.

7 (a) V. Castelletto, I. W. Hamley, R. A. Hule and D. J. Pochan, Angew. Chem., Int. Ed. Engl., 2009, 48, 2317-2320; (b) J. Adamcik, V. Castelletto, I. W. Hamley and R. Mezzenga, Angew. Chem., Int. Ed. Engl., 2011, 50, 5495-5498; (c) E. T. Pashuck and S. I. Stupp, J. Am. Chem. Soc., 2010, 132, 8819-8821.

8 (a) P. G. Degennes, C. R. Acad. Sci., Ser. IIc: Chim., 1987, 304, 259-263; (b) J. V. Selinger, F. C. MacKintosh and J. M. Schnur, Phys. Rev. E: Stat. Phys., Plasmas, Fluids, Relat. Interdiscip. Top., 1996, 53, 3804-3818; (c) J. V. Selinger, M. S. Spector and J. M. Schnur, J. Phys. Chem. B, 2001, 105, 7157-7169.

9 Z. Huang, S. K. Kang, M. Banno, T. Yamaguchi, D. Lee, C. Seok, E. Yashima and M. Lee, Science, 2012, 337, 15211526.

10 (a) L. O. Tjernberg, J. Naslund, F. Lindqvist, J. Johansson, A. R. Karlstrom, J. Thyberg, L. Terenius and C. Nordstedt, J. Biol. Chem., 1996, 271, 8545-8548; (b) M. J. Krysmann, V. Castelletto, A. Kelarakis, I. W. Hamley, R. A. Hule and D. J. Pochan, Biochemistry, 2008, 47, 4597-4605; (c) I. W. Hamley, Chem. Rev., 2012, 112, 5147-5192.

11 M. J. Krysmann, V. Castelletto and I. W. Hamley, Soft Matter, 2007, 3, 1401-1406.

12 A. Dehsorkhi, I. W. Hamley, J. Seitsonen and J. Ruokolainen, Langmuir, 2013, 29, 6665-6672. 
13 (a) W. K. Surewicz, H. H. Mantsch and D. Chapman, Biochemistry, 1993, 32, 389-394; (b) A. Barth, Biochim. Biophys. Acta, Bioenerg., 2007, 1767, 1073-1101.

14 H. LeVine, Methods in Enzymology, ed. R. Wetzel, Academic Press, San Diego, 1999, vol. 309, pp. 274-284.

15 (a) H. LeVine, Protein Sci., 1993, 2, 404-410; (b) I. W. Hamley, Angew. Chem., Int. Ed., 2007, 46, 8128-8147.

16 (a) M. R. H. Krebs, E. H. C. Bromley and A. M. Donald, J. Struct. Biol., 2005, 149, 30-37; (b) R. Khurana, C. Coleman, C. Ionescu-Zanetti, S. A. Carter, V. Krishna, R. K. Grover,
R. Roy and S. Singh, J. Struct. Biol., 2005, 151, 229-238; (c) W. S. Childers, A. K. Mehta, K. Lu and D. G. Lynn, J. Am. Chem. Soc., 2009, 131, 10165-10172.

17 T. E. Creighton, Proteins. Structures and Molecular Properties, W.H. Freeman, 1993.

18 A. Barth and C. Zscherp, Q. Rev. Biophys., 2002, 35, 369430.

19 L. Milanesi, J. P. Waltho, C. A. Hunter, D. J. Shaw, G. S. Beddard, G. D. Reid, S. Dev and M. Volk, Proc. Natl. Acad. Sci. U. S. A., 2012, 109, 19563-19568. 